\title{
Machine Vision for Airport Runway Identification
}

Matthew Schubertabc, Andrew J. Moore ${ }^{\mathrm{a} *}$, Chester Dolph $^{\text {ad }}$, Glenn Woodellae

aComputational Vision Lab, NASA Langley Research Center, Hampton, VA, USA bNational Institute of Aerospace, Hampton, VA, USA 'Christopher Newport University, Newport News, VA, USA dVision Lab, Old Dominion University, Norfolk, VA, USA eScience Systems and Applications, Inc., Hampton, VA, USA 


\section{Acknowledgements}

- NASA Atmospheric and Environmental Safety Technologies program

- NASA Safe Autonomous Systems Operations program

- Rockwell Collins 


\section{Significance}

Incorrect runway problem

Over 500 incidents since 1999 (FAA)

No injuries or fatalities, but close calls

Geolocation technologies

Plane lands at wrong West Virginia Airport; No one injured

Boeing 747 lands at wrong airport in Kansas

Radio (e.g. VOR)

GPS

Gyros (inertial)

Human vision

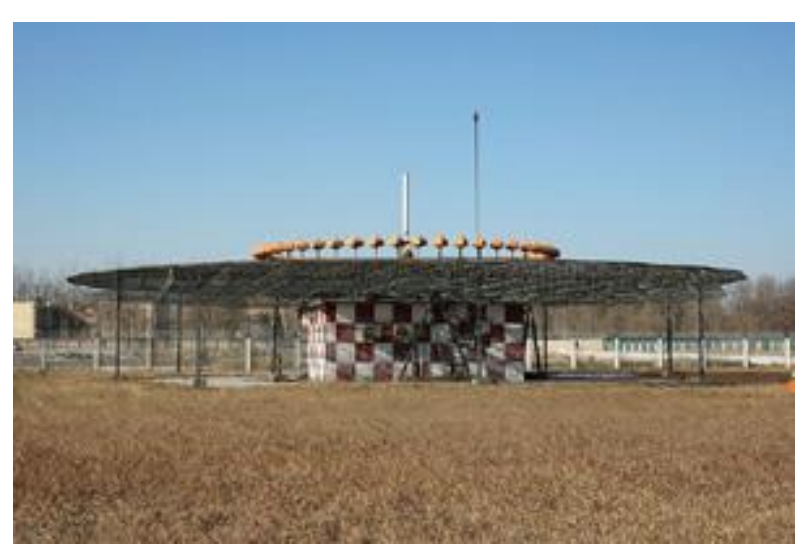

Source: FAA 


\section{Possible: Warning of Incorrect Landing}

Night Approaches at 3 Airports - Very Strong Signal



FRA 25L

Frankfurt DE

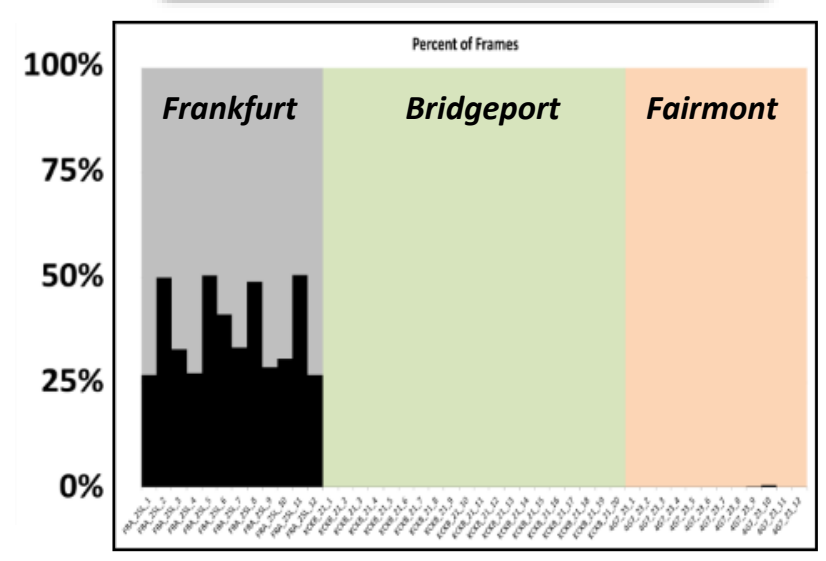

9477-14 Machine Vision for Runway ID

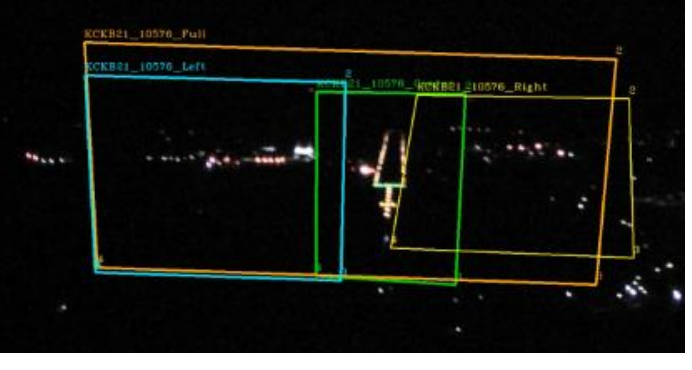

KCKB 21

Bridgeport WV

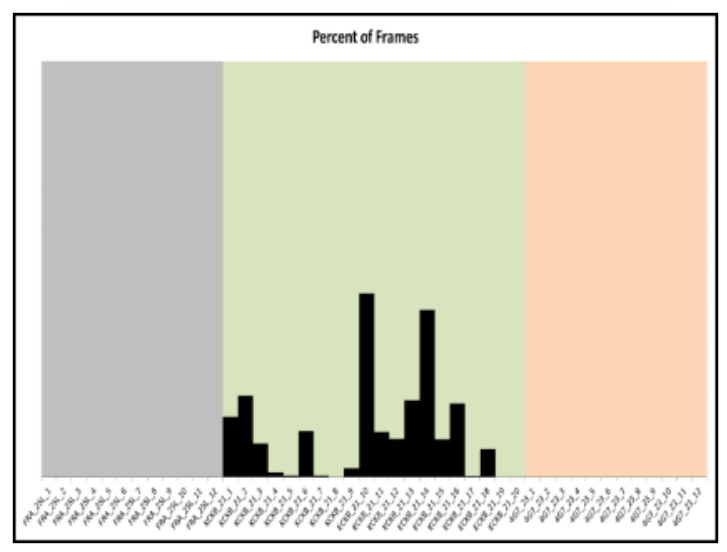

Optical Pattern Recognition XXVI
Source: NASA

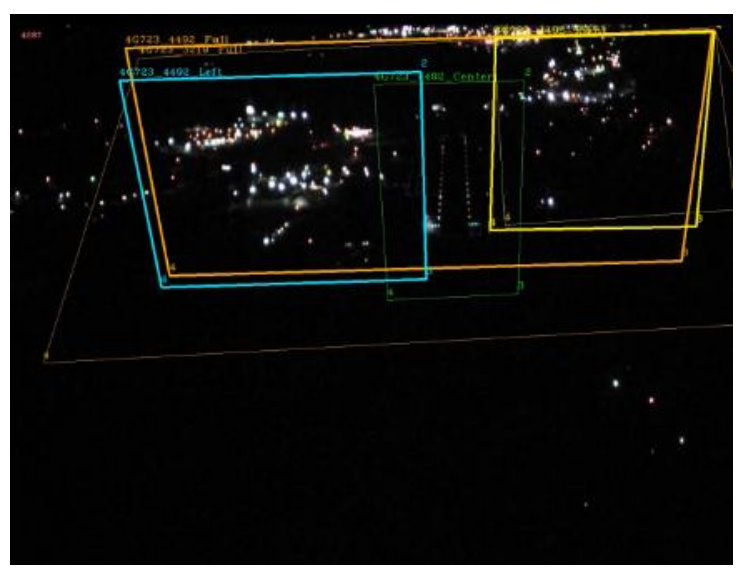

$4 \mathrm{G} 723$

Fairmont WV






\section{Hypothesis assumptions and predictions}

For rigid objects and fixed scenes, current machine vision technology is capable of identifying imagery rapidly and with specificity over a modest range of camera viewpoints and scene illumination.

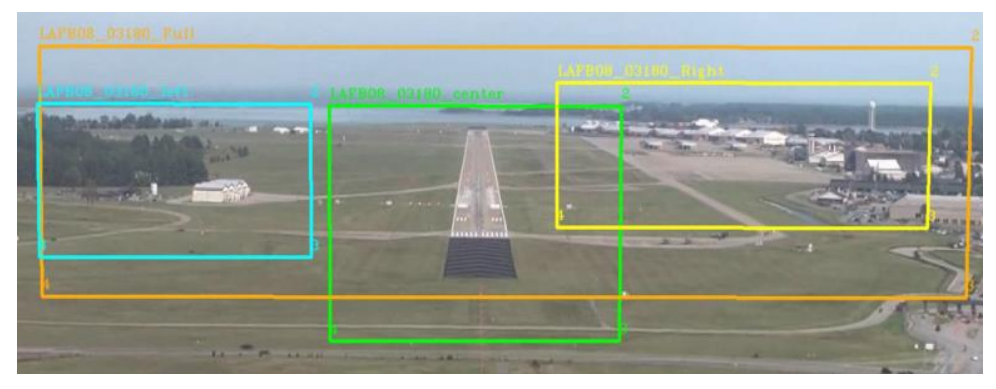

Source: NASA
Rigid objects

Fixed scenes

Modest viewpoint change

Modest illumination change
Runway video is recent - no major construction

No snow cover, full foliage

Variation due to atmospheric turbidity rain and fog in some approaches

Reject "cross-ways flyover" data

No dawn/dusk flights: two illumination variants (day and night). 


\section{Hypothesis assumptions and predictions}

For rigid objects and fixed scenes, current machine vision technology is capable of identifying imagery rapidly and with specificity over a modest range of camera viewpoints and scene illumination.

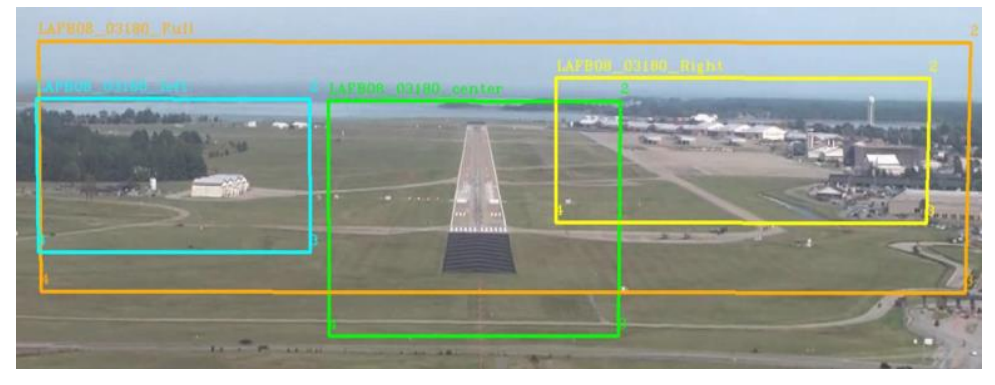

Source: NASA
Rapid

Specific

"Stretch" prediction

Repeatable
Only for
Yes: 10fps for most tests

Yes: if the statistics are done correctly

- same illumination (day vs. night)

- same sensor is used

- visibility was good 


\section{Data Prep}

Raw videos

resolution: $\quad$ visible $-1000 \times 700$ resolution or better infrared - 640x480

frame rate: $\quad 30$ frames per second

Flights rejected

perpendicular flyovers

very distant views

several from Google Earth

several from simulators 


\section{9 videos, 9 locations}

\begin{tabular}{|c|c|c|c|c|c|c|}
\hline Approach Video & Runway & Band & Time & Weather & $\begin{array}{l}\text { Way } \\
\text { points }\end{array}$ & $\begin{array}{c}\text { Refs/ } \\
\text { waypoint }\end{array}$ \\
\hline 4G7_23_night & 1 & & night & clear & 3 & $4,4,4$ \\
\hline EDDF_25L_night & 2 & & night & clear & 3 & $4,4,4$ \\
\hline KAVC_01_fog_S & $3 *$ & & day & fog/rain & 3 & $2,2,2$ \\
\hline KAVC_01_fog_SE & $3 *$ & & day & fog/rain & 2 & 1,1 \\
\hline KCID_27_LW & $4^{*}$ & LW & day & fog/snow & 6 & $4,4,4,4,4,4$ \\
\hline KCID_27_SW & $4 *$ & SW & day & fog/snow & 6 & $4,4,4,4,4,4$ \\
\hline KCID_27_VIS & $4^{*}$ & & day & fog/snow & 8 & $4,4,4,4,4,4,4,4$ \\
\hline KCID_27_LW_night & $4 *$ & LW & night & clear & 5 & $4,4,4,4,4$ \\
\hline KCID_27_SW_night & $4^{*}$ & SW & night & clear & 4 & $4,4,4,4$ \\
\hline KCID_27_VIS_night & $4 *$ & & night & clear & 5 & $4,4,4,4,4$ \\
\hline KCKB_21_night & 5 & & night & clear & 5 & $4,4,4,4,4$ \\
\hline KLAS_25L_LW_night & $6 *$ & LW & night & clear & 4 & $4,4,4,4$ \\
\hline KLAS_25L_SW_night & $6 *$ & SW & night & clear & 7 & $4,4,4,4,4,4,4$ \\
\hline KLAS_25L_VIS_night & $6 *$ & & night & clear & 6 & $4,4,4,4,4,4$ \\
\hline KLFI_08_S & $7^{*}$ & & day & clear & 3 & $4,4,4$ \\
\hline KLFI_08_W & $7 *$ & & day & clear & 4 & $4,4,4,4$ \\
\hline KLFI_08_fog_NW & $7 *$ & & day & fog/rain & 3 & $1,1,3$ \\
\hline KLFI_26_rain & 8 & & day & fog/rain & 5 & $4,4,4,4,4$ \\
\hline KPHF_25 & 9 & & day & clear & 3 & $4,4,4$ \\
\hline Total & 9 & & & & 85 & 321 \\
\hline
\end{tabular}




\section{Waypoints and Epochs}

Waypoint Waypoint Waypoint Waypoint Waypoint
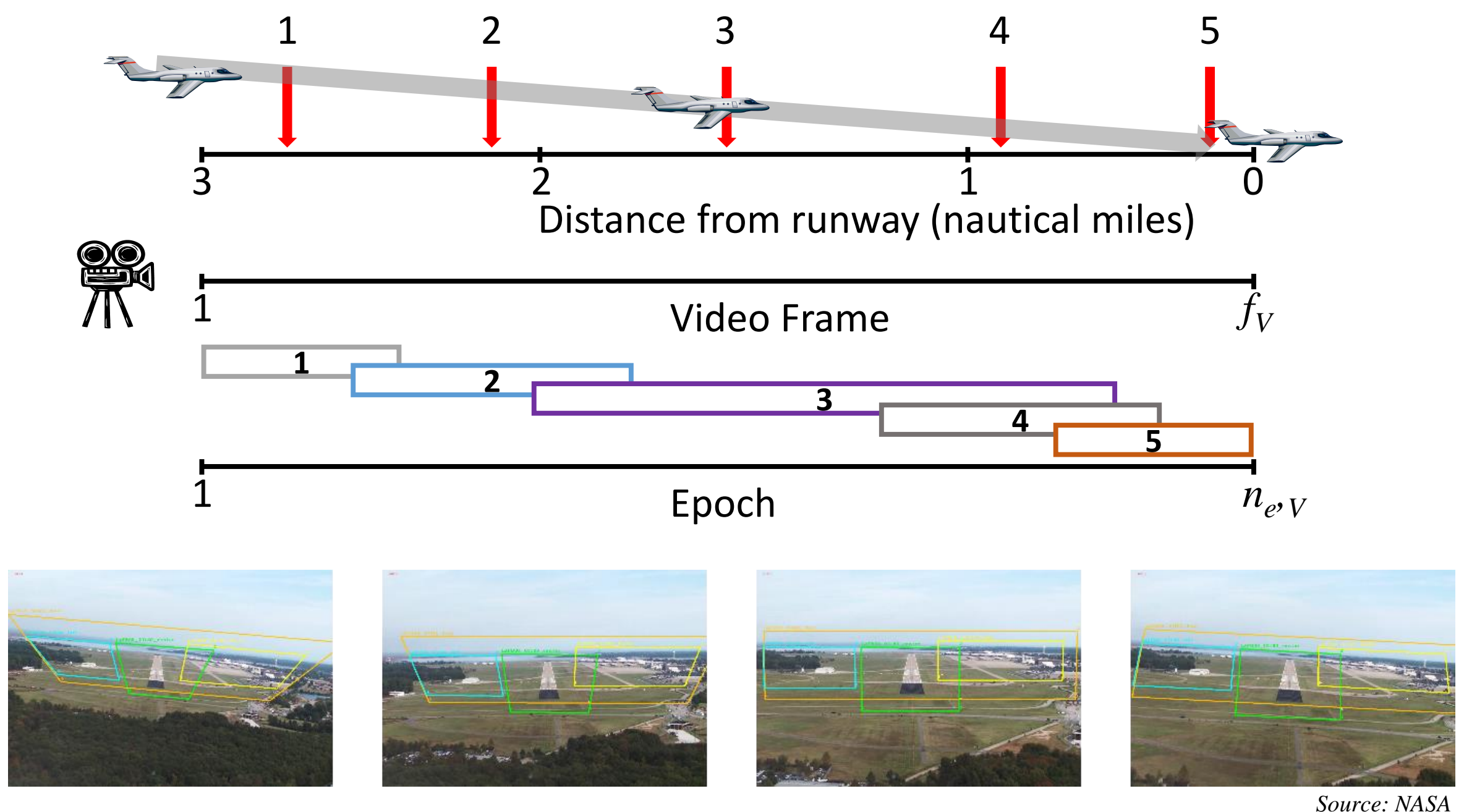


\section{Bayesian Probability Model}

$P\left(e \mid R_{i}\right)=\frac{P\left(R_{i} \mid e\right) P(e)}{P\left(R_{i}\right)} \quad P(e)=\frac{1}{n_{e}}$

Precompute: $\quad P\left(R_{i} \mid e\right)=\frac{\sum_{f=1}^{f_{e}} M_{i, f}}{f_{e}}$

$$
P\left(R_{i}\right)=\frac{\sum_{e=1}^{n_{e}} P\left(R_{i} \mid e\right)}{n_{e}}
$$

Compute on the fly:

$$
P\left(e_{t}\right)=\left[1-\prod_{i=1}^{k}\left(1-P\left(e \mid R_{i}\right) \cdot M_{i, t}\right)\right]
$$

KCID_27_SW_night

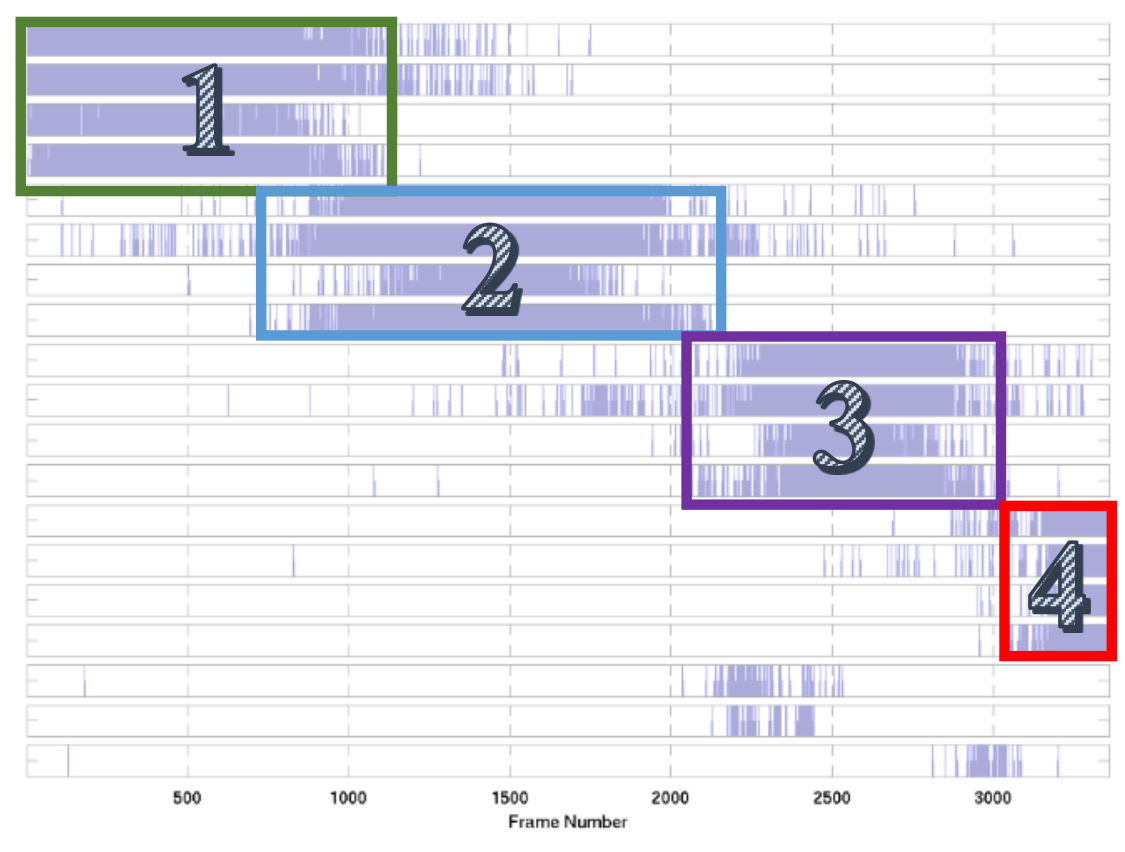

Epoch $e$ is a proxy for location; location is unknown by default. "Unless one of the images I contain is matched in this frame, my probability is zero. Every match of one of the images that I contain increases my probability towards one." 


\section{Bayesian Probability Model}

Source: NASA

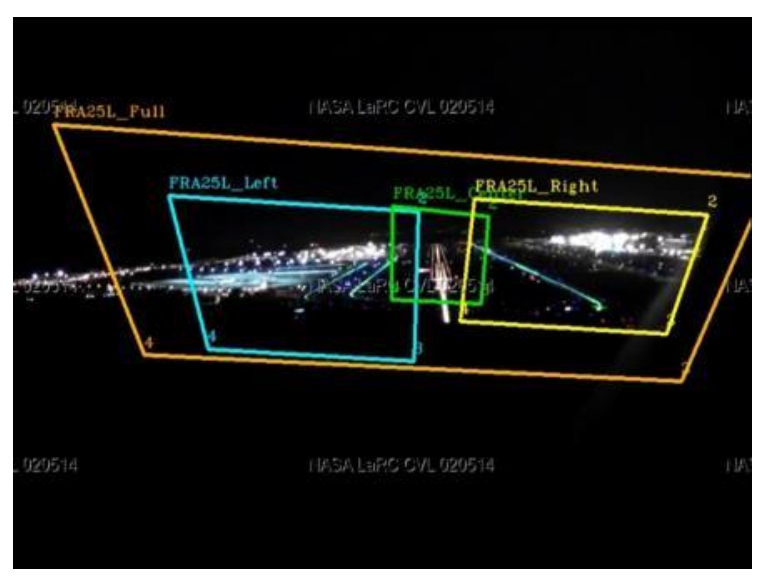

FRA 25L

Frankfurt DE

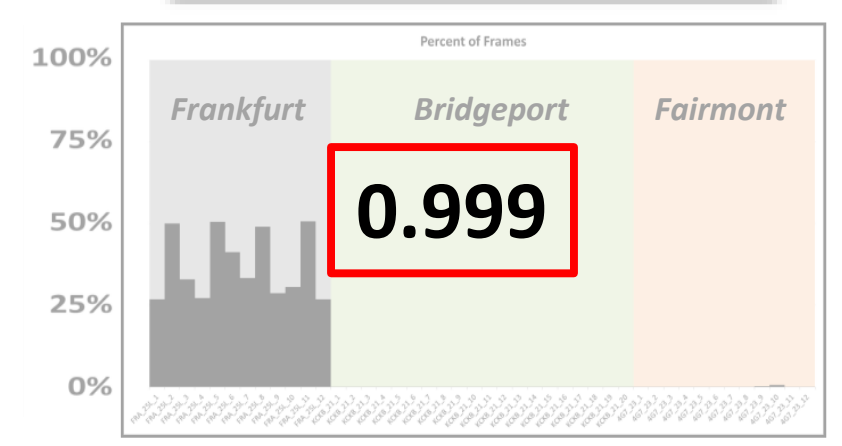

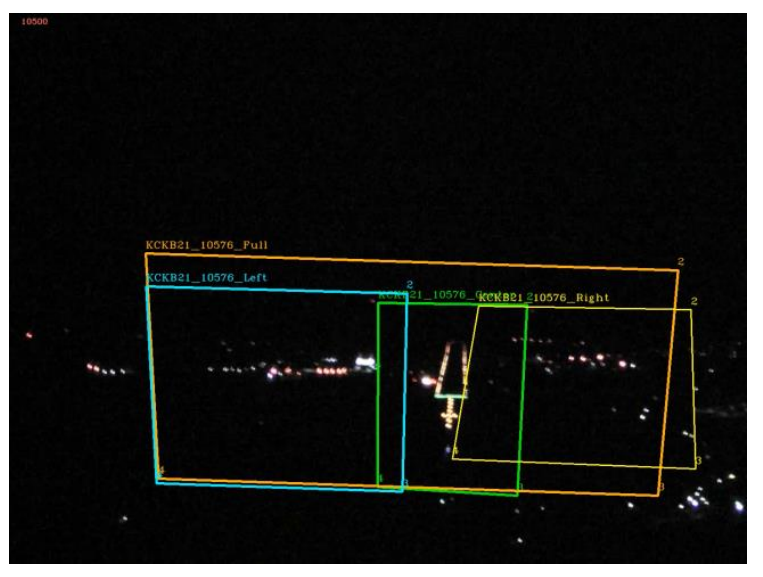

KCKB 21

Bridgeport WV

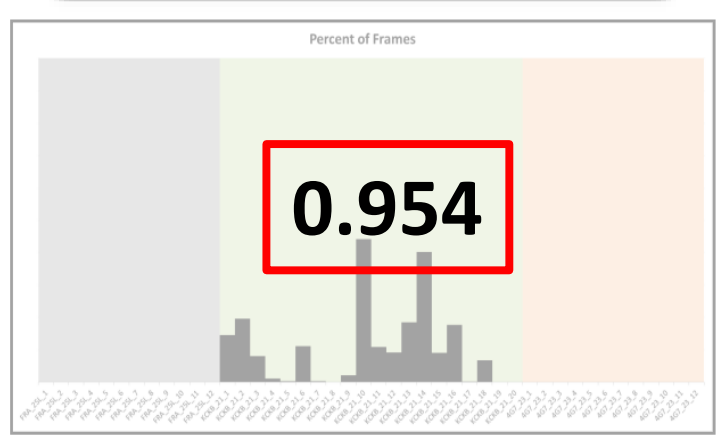

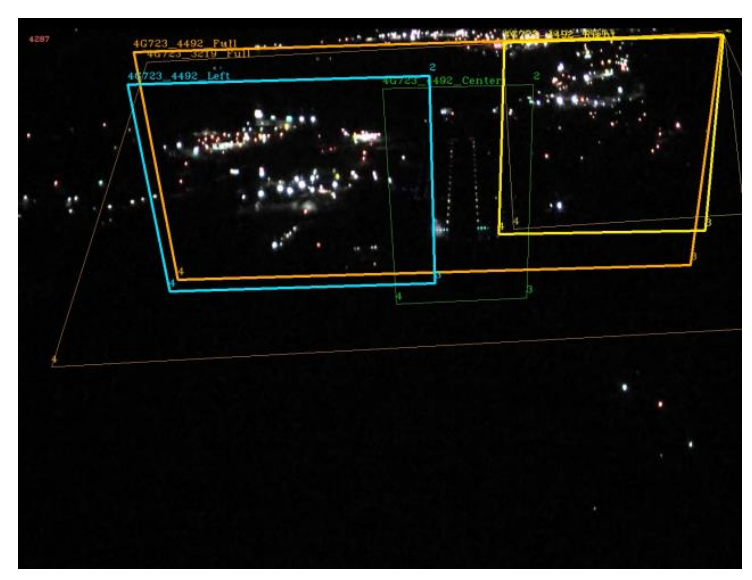

$$
4 G 723
$$

Fairmont WV






\section{How to Represent Concisely?}
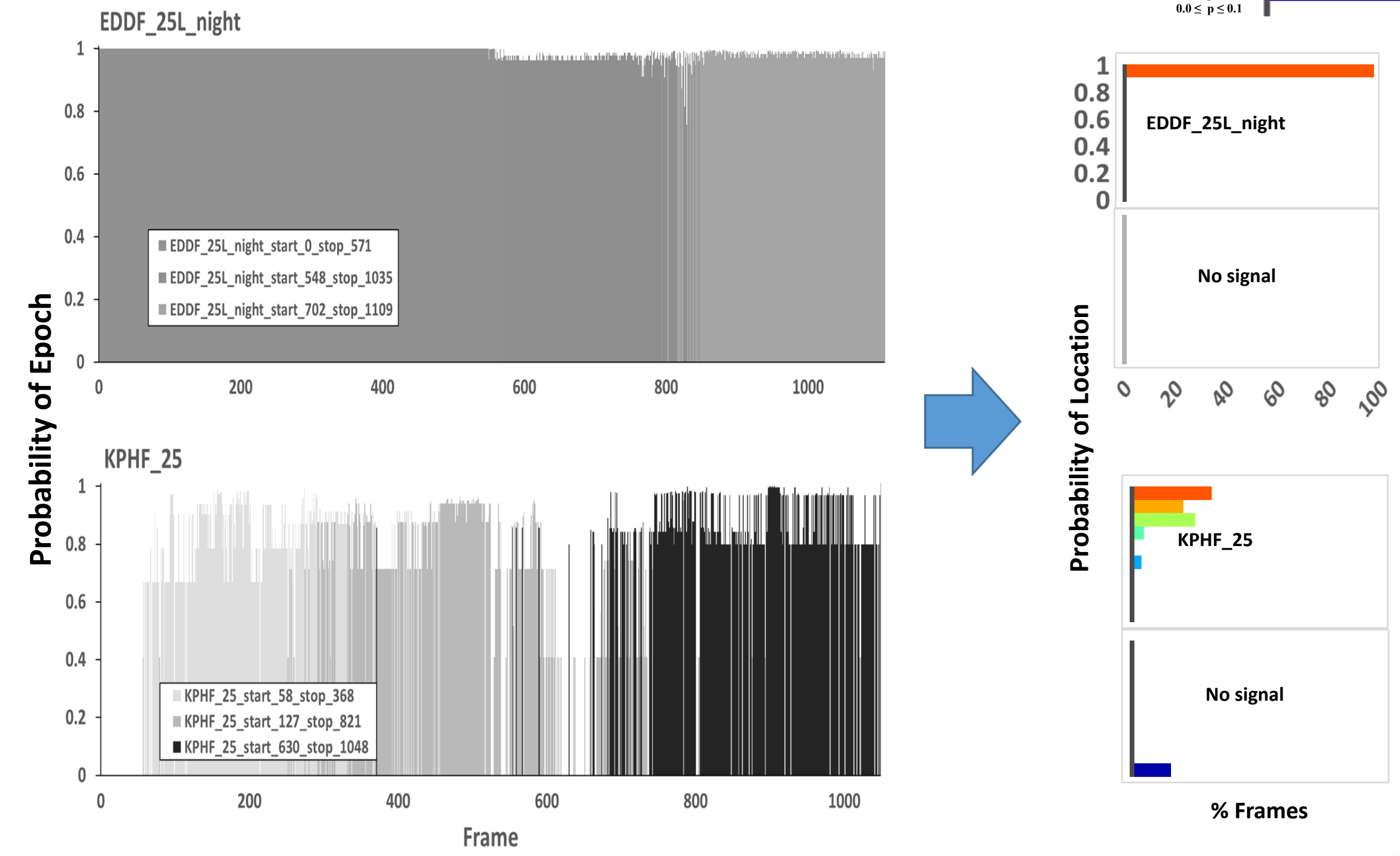


\section{Results: Specificity}

\section{Estimated vs. True Location}

Approach

4G7_23_night EDDF_25L_night KAVC_01_fog_S KAVC_01_fog_SE KCID_27_LW KCID_27_SW KCID_27_VIS KCID_27_LW_night KCID_27_SW_night KCID_27_VIS_night KCKB_21_night KLAS_25L_LW_night KLAS_25L_SW_night KLAS_25L_VIS_night KLFI_08_S KLFI_08_W KLFI_08_fog_NW KLFI_26_rain KPHF_25
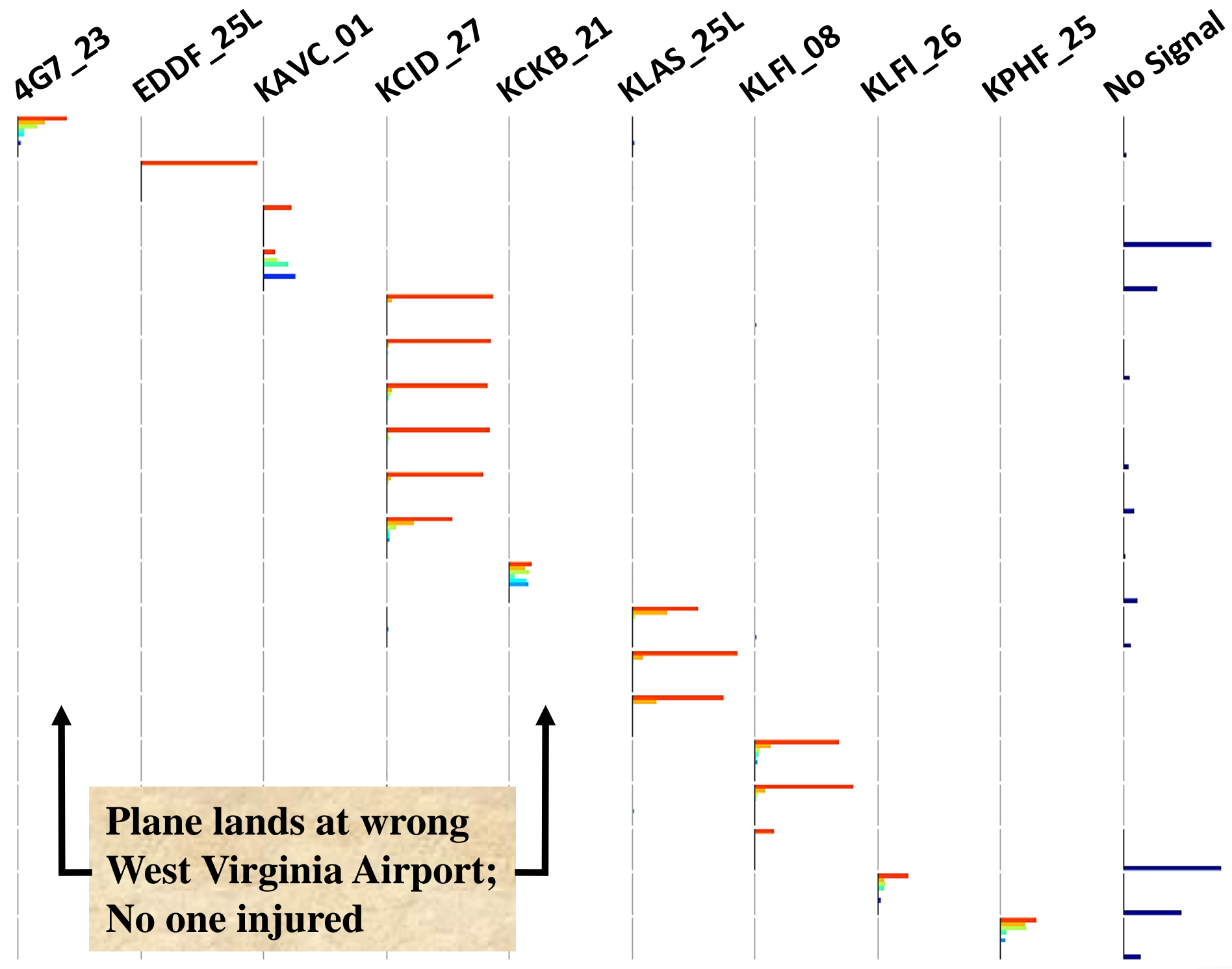


\section{Results: Specificity (High Pass)}

Estimated vs. True Location

Approach



4G7_23_night

EDDF_25L_night

KAVC_01_fog_S

KAVC_01_fog_SE

KCID_27_LW

KCID_27_SW

KCID_27_VIS

KCID_27_LW_night

KCID_27_SW_night

KCID_27_VIS_night

KCKB_21_night

KLAS_25L_LW_night

KLAS_25L_SW_night

KLAS_25L_VIS_night

KLFI_08_S

KLFI_08_W

KLFI_08_fog_NW

KLFI_26_rain

KPHF_25

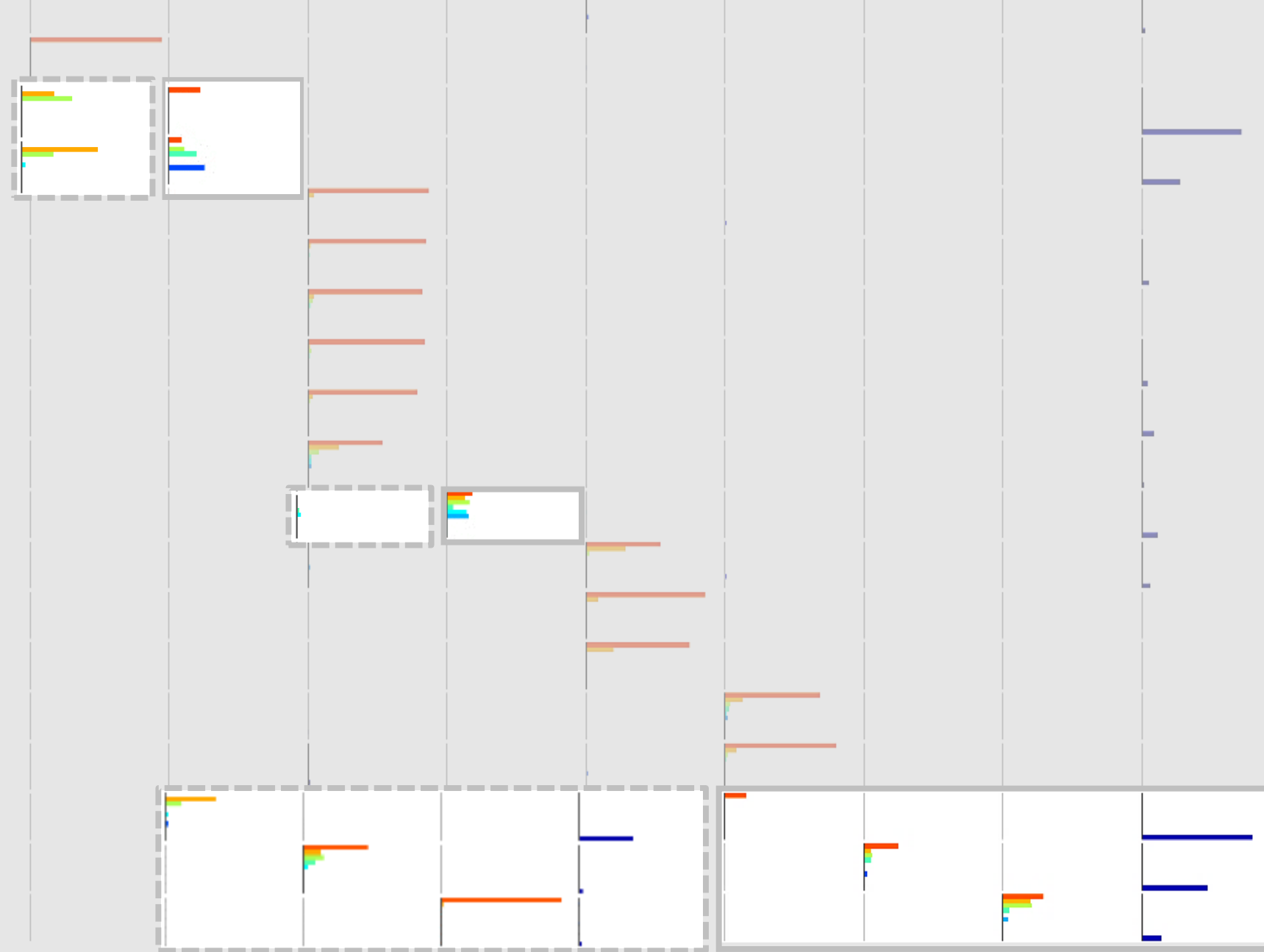




\section{Results: Repeatability}

Estimated vs. True Approach

Approach

KAVC_01_fog_S

KAVC_01_fog_SE

KCID_27_LW

KCID_27_SW

KCID_27_VIS

KCID_27_LW_night

KCID_27_SW_night

KCID_27_VIS_night

KLAS_25L_LW_night

KLAS_25L_SW_night

KLAS_25L_VIS_night

KLFI_08_S

KLFI_08_W

KLFI_08_fog_NW






\section{Cross-matching between visible and SWIR}

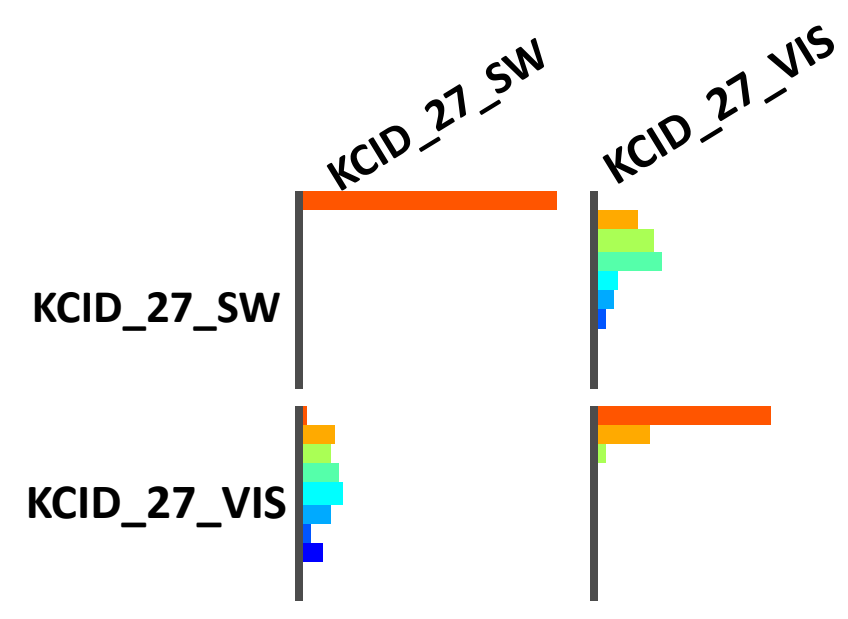

KCID_27_SW

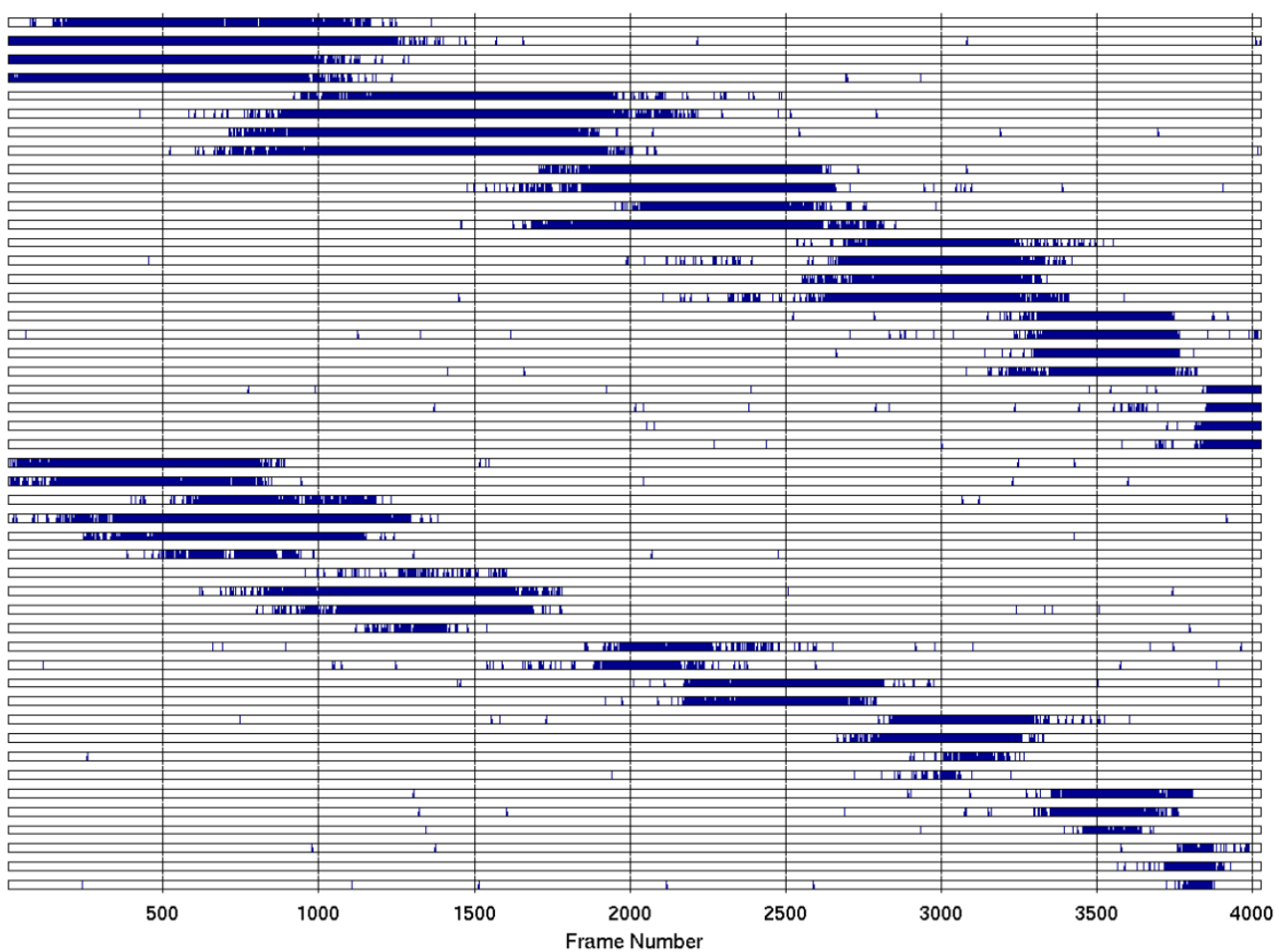




\section{Conclusions}

Rapid

Specific

Repeatable $\checkmark *$
For rigid objects and fixed scenes, current machine vision technology is capable of identifying imagery rapidly and with specificity

over a modest range of camera viewpoints and scene illumination.

*

Robust if illumination (day vs. night) and sensor are the same.

$*$

Both specificity and repeatability degraded in poor weather.

Surprise result: cross-sensor repeatability (visible <-> SWIR)

Geolocation via real-time comparison of cockpit video to a database is feasible, as long as

- the database contains imagery from the same time of day and

- the weather is clear at the time of the flight.

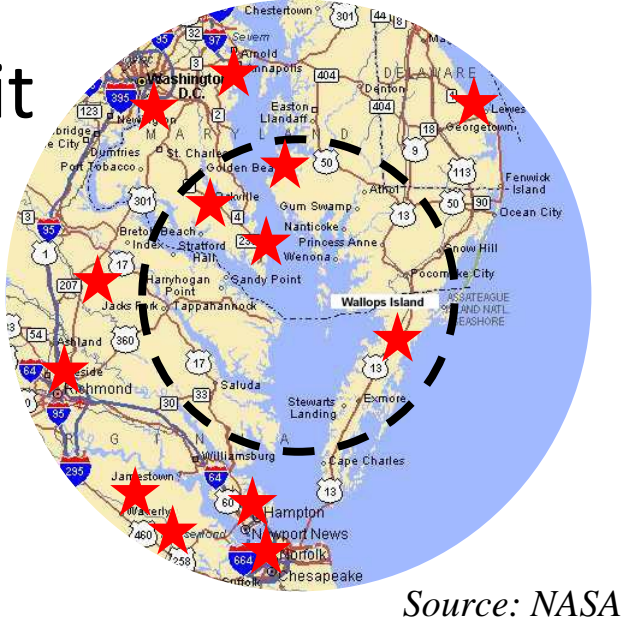




\section{Conclusions}

\section{Applications}

Geolocation validation and verification is already possible:

GPS - most of the approach

Inertial Guidance - all of the approach

Machine vision may (for clear flight conditions):

supplement these or

provide the same capability at lower cost,

though the certification cost is a significant barrier to adoption.

The limitations of machine vision geolocation in poor weather ensure that it cannot be the sole backup navigational technology.

Tests of its effectiveness with weather-penetrating sensors could overcome this limitation.

Anticipated over 15 years ago. Machine vision is now capable and compact enough to pursue this. 


\section{Backup}




\section{Literature Review (abbreviated)}

\section{Edge detection}

Huertas et al. in 1990 used localized edge detection and thresholding to outline runways and create location-specific image templates for use in an expert system [12; see also 16].

\section{Hough transform}

Fleming and his collaborators reviewed the literature through 2004 [13] (including work that harnessed a key innovation, the Hough transform) and applied the Hough transform to runway imagery, using stereo ranging to estimate the airplane viewpoint and landing distance [14]. Independently and contemporaneously, Shang and Shi [15] took a similar approach, using monocular perspective analysis instead of stereo analysis to estimate the landing geometry.

\section{Primitive learning}

In his Master's thesis, Zongur [17] added a machine learning layer to previously applied techniques to recognize airports from orbital imagery.

\section{Modern machine vision}

Medioni and his colleagues at USC and Honeywell [18] used a new class of robust feature detector [2] and homographic perspective transformation to track runways in flight video; with image stabilization and image differencing they could determine if a runway was free of hazardous objects during the landing approach. Their application of the scale-invariant SIFT front end in 2009 represents a qualitative improvement in robustness in machine vision for aeronautics -- we use a performance-optimized variant (SURF [23]) in this study.

\section{Next generation}

A team at EPFL in 2014 applied methods at the current state of the art of machine vision to determine the boundaries of alternate landing sites such as agricultural fields in real time [20]. 


\section{Epochs are containers of probability}

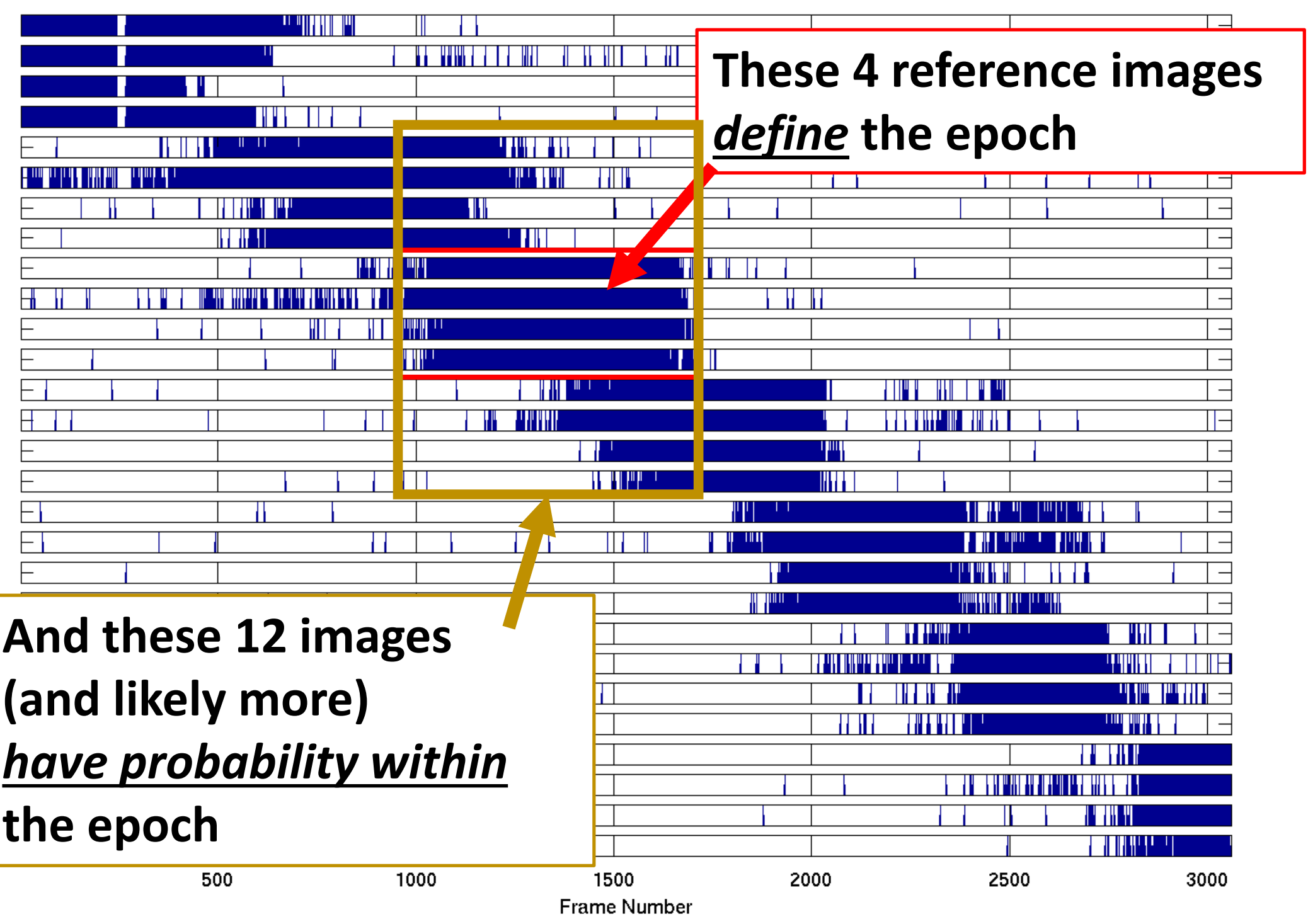




\section{Conclusions}

Aim of this study: explore the limitations of current machine vision technology, as applied to airborne geolocation, with realistic runway approach video taken in a variety of flight conditions. Our expectations:

- Rapid results

- Specific results

- Stability only for rigid objects

- Stability only for fixed scenes

- Stability only with modest change in illumination

- Stability only with modest change in viewpoint

Overall, the results met these expectations. 


\section{Conclusions}

\section{Speed, Stability}

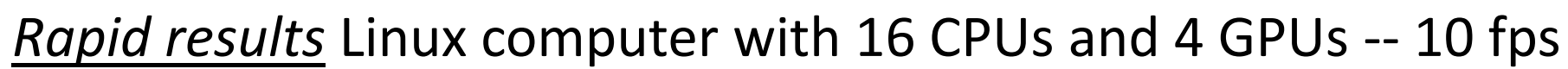

Specific results As long as the flight conditions did not depart from the key constraints (rigid objects, fixed scenes, modest variation in illumination and viewpoint), location was determined with excellent specificity.

Rigid objects This constraint was maintained strictly throughout the study, in the sense that a clear runway or a patch of landscape viewed from the air is a fixed object.

For example, we did not test cases with obstacles on the runway, or cases in which heavy winds cause trees in the landscape to move noticeably. 


\section{Conclusions}

\section{Scene Variation}

The "fixed scene" constraint was varied in two ways.

1. In clear conditions we allowed minor changes within the scene: the moving vehicles. They had no discernable effect on the results.

2. A change in air turbidity is in essence a scene change. Four video approaches in fog/rain. Specificity was superb even with this violation of the fixed scene constraint. However, the constancy of specificity throughout the flight, was degraded. Contrast:

- EDDF_25L_night -- strong location nearly $100 \%$ of frames

- KAVC_01_fog_S -- strong location only $20 \%$ of frames

EDDF_25L_night

KAVC_01_fog_S

At first we suspected that constancy of location was degraded due to methodology, i.e., poor reference imagery choice or low waypoint count. But, KAVC 01 fog $S$ and KAVC 01 fog SE seem to disprove this.

KAVC_01_fog_SE -- fewer waypoints and reference images but its location correct for over $50 \%$ of the approach (albeit with less than $90 \%$ confidence for much of that time).

\begin{tabular}{|l|c|c||c|c|}
\hline Approach Video & $\begin{array}{c}\text { Way } \\
\text { points }\end{array}$ & $\begin{array}{c}\text { Refs/ } \\
\text { waypoint }\end{array}$ & Epochs & $\begin{array}{c}\text { Refs defining } \\
\text { epoch }\end{array}$ \\
\hline KAVC_01_fog_S & 3 & $2,2,2$ & 3 & $1,1,2$ \\
\hline KAVC_01_fog_SE & 2 & 1,1 & 2 & 1,1 \\
\hline
\end{tabular}

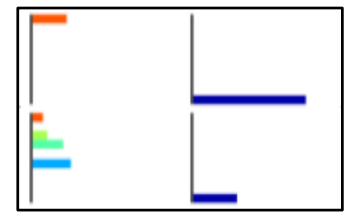

We did not use transmissometers to quantify visibility, and cannot identify a root cause. 


\section{Conclusions \\ Illumination change}

The "modest illumination change" constraint was maintained strictly throughout the study. Only mid-day and nighttime approach video was available.

Shadows: A change in shadows early or late in the day is an example of illumination change that violates the fixed scene assumption; more data is required to assess the fragility of machine vision due to shadows.

Diffuse vs. direct sunlight: What about, say, direct vs. diffuse sunlight on a foggy day?

We tested overcast conditions only. We assume that air turbidity is a stronger effect but cannot prove it with this data. 


\section{Conclusions Modest viewpoint change}

The waypoint methodology ensures viewpoint constancy.

Simple conclusion: machine vision fails as expected when the viewpoint is changed greatly. For example, epoch locations from early in an approach have zero reported probability late in an approach.

Stronger conclusion: runway recognition with machine vision is effective with modest viewpoint changes.

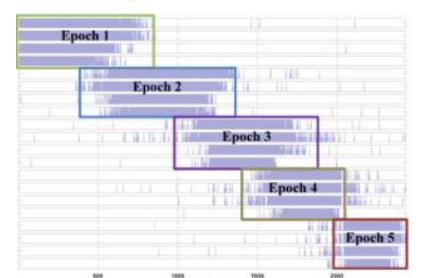

Impörtant exception:

a) the continuity of reported location is less variable for a large aircraft (Boeing 747, at top)

than a small aircraft (Cessna, at bottom)

b) the camera viewpoint in recordings from a small airplane is prone to sudden changes in pitch, yaw or roll presumably due to wind shifts during the flight. for $15-30$ seconds.
Example - KMEM_09_IR_night. What is remarkable is the time span of the positive result: reference images from a single 33 millisecond time sample (a single frame) produce a correct result

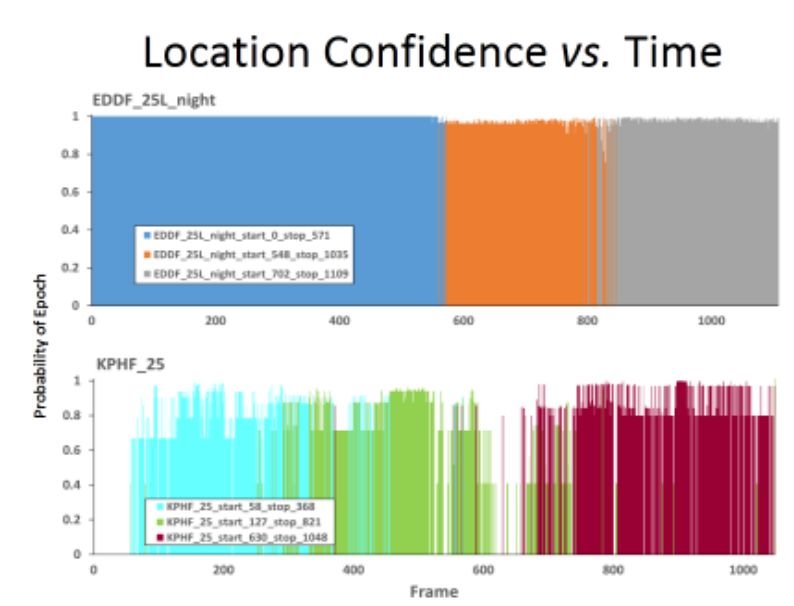

Since everything is recomputed for each frame, this does not arise from compute latency. Looking at the original video for KPHF 25 and other Cessna recordings, we observed that the image sensor does not respond instantly to a sudden viewpoint change. Streaking, smearing and other frame readout artifacts are evident with each jerk. We conclude that a sensor with a higher frame rate, a faster pixel readout response, or frame-shielded design is needed to eliminate this effect. This sensor constraint should be observed in General Aviation and in small UAV applications. 


\section{Conclusions}

\section{Repeatability}

Clear daylight conditions: Machine vision can repeatedly locate a runway.

Clear night conditions: We expect that repeatability is possible but can't prove it.

Fog or rain: Violate the fixed scene constraint and wipe out repeatability, even between two foggy approaches.

Across sensors and across time of day: Need more data to answer this.

Only simulator data was available to test across sensors (visible/SWIR) and across time of day

Because of these shortcomings, we cannot say that repeatability is beyond the capabilities of machine vision as the sensor or time of day is changed.

\section{Simulator Pluses:}

- Fidelity is sufficient to produce high specificity even with $640 \times 480$ resolution.

- Free from the sudden viewpoint changes as observed in the Cessna flights.

- Geodetics are superb - landscape features are identical across sensor types and times of day.
Simulator Minus:

Landscape features are not photorealistic.

- Nighttime light placement seems arbitrary as perspective increases toward the vanishing point.

- Ground features such as buildings are generally "flattened and painted" onto the ground. 


\section{Conclusions}

\section{Applications}

Geolocation validation and verification is already possible:

GPS - most of the approach

Inertial Guidance - all of the approach

Machine vision may

supplement these, or

provide the same capability (for some flight conditions)

at lower cost,

though the effort to certify machine vision to the level of inertial technology is a significant barrier to adoption.

With visible and SWIR sensor inputs, the limitations of machine vision geolocation in poor weather ensure that it cannot be the sole backup navigational technology.

Tests of its effectiveness with weather-penetrating sensor inputs are required to overcome this limitation. Anticipated over 15 years ago. 


\section{Conclusions}

\section{Operational feasibility}

An aircraft with

- an onboard image sensor,

- modest computing power, and

- a database of reference images and probabilities

can geolocate as long as visibility is clear and the database

- includes imagery from various of times of day,

computation time will increase linearly with database size

- covers the geographical areas likely to be encountered, and

- contains imagery of the same sensor type as the onboard sensor.

We expect that the imagery database must also:

1. include seasonal variations such as snow cover, wet vs. dry pavement, and full vs. sparse foliage, and

2. be reasonably current. Minor scene changes did not degrade results, and we expect that similarly minor changes (traffic cones, commercial signage, and cell towers) will not do so either. Major changes, such as new roadways and buildings will violate the fixed scene assumption. 